\title{
Eosinophilic granuloma of spine in adults: a case report and review of literature
}

\author{
PK Reddy ${ }^{1}$, PSSV Vannemreddy ${ }^{1}$ and A Nanda*,1 \\ ${ }^{1}$ Department of Neurosurgery, Louisiana State University Medical Center, Shreveport, Louisiana, USA
}

A rare case of eosinophilic granuloma in an adult is reported. Eosinophilic granuloma (EG) is a lesion observed more frequently in adults. CT and MRI showed a lytic lesion of the T11 vertebral body. A transpedicular excisional biopsy of the lesion revealed EG. Spinal EG in adults is rare and differs from the childhood disease by the spinal level involvement. Vertebra plana, a condition of spondylitis in which the body of the vertebra is reduced to a sclerotic disc, is not a roentgenographic feature in the 14 cases reported in literature. It should be included in the differential diagnosis of the solitary lytic lesion of vertebrae in adults.

Spinal Cord (2000) 38, $766-768$

Keywords: eosinophilic granuloma; adult spine; vertebra plana; Langerhans' cell histiocytosis

\section{Introduction}

Eosinophilic granuloma (EG) is one element in the protean spectrum of diseases associated with Langerhans' cell histiocytosis X. ${ }^{1}$ Hand-Schuller-Christian disease and Letterer-Siwe disease constitute the acute and subacute forms of histiocytosis $\mathrm{X}$, while eosinophilic granuloma is the most common and benign form. This idiopathic lytic disease of bone usually occurs in children and adolescents. Although EG can involve any bone, it is most commonly found in the flat bones of the cranium, mandible, ribs, clavicle, pelvis and long bones of extremities. ${ }^{2}$

A solitary cranial lesion is the most common presentation. While spinal involvement by $E G$ is uncommon at any age, it is a distinct rarity in adults and only 14 adult cases have been reported in the English language literature. The classical presentation of EG in children involves the thoracic spine and appears as a 'flattened vertebra' or vertebra plana. However, in adults, the presentation has certain differences.

\section{Case report}

A 47-year-old hypertensive white man presented with a 6-week history of low back pain. Pain was constant, radiated anteriorly to the umbilical region and had no aggravating or relieving factors. The patient denied any bowel or bladder problems. No numbness or tingling of the lower extremities and no history of weight loss or blood in stool was noted in the patient, nor was there any motor deficit in the upper or lower extremities. The patient had no constitutional symp-

*Correspondence: A Nanda, Louisiana State University Medical Center-Shreveport, PO Box 33932, 1501 Kings Highway, Shreveport, LA 71130-3932, USA toms. On neurological examination, cranial nerves II through XII were intact. Deep tendon and superficial reflexes were intact and symmetrical. There was some impairment of sensation in the distribution of T11 to T12 dermatomes. Plain X-ray of the thoracic spine showed a destructive lesion of the T11 vertebral body. A CT scan of this region of the thoracic spine revealed a localized lytic lesion in the vertebral body with sclerotic margins. On MR imaging, the lesion had mixed intensities on $\mathrm{T} 1$, hyperintensity on $\mathrm{T} 2$, and dense rim enhancement on contrast administration (Figure 1a,b). A bone scan confirmed this to be a solitary hot spot. A preoperative diagnosis of metastasis was made and a CT-guided aspiration was inconclusive. A transpedicular excision of the mass and fusion of T11-T12 was performed. The lesion was predominantly cystic and contained a white amorphous material. Frozen sections were consistent with inflammatory tissue, and no tumor was seen. Gram staining, AFB smear, bacterial and fungal cultures were negative. On histological examination, the tissue showed features of eosinophilic granuloma with aggregates of Langerhans' histiocytes (Figure 2). The patient tolerated the surgery well with no postoperative neurological deficits. A follow-up MRI 1 year later showed no residue or recurrence.

\section{Discussion}

EG is the benign form of Langerhans' cell histiocytosis, a disease of the reticuloendothelial system. In 1925, Calve described two children with roentgenographic evidence of complete collapse of the vertebral body with preservation of disc spaces. He believed this to be an osteochondritis of the spine. In 1927, Buchman suggested the name vertebra plana for this roentgeno- 


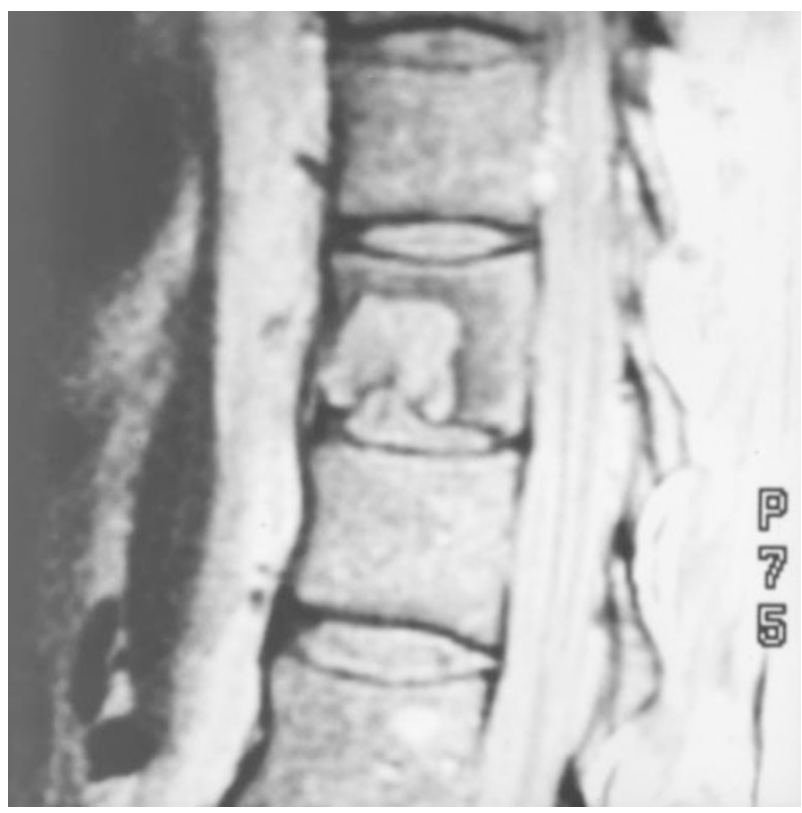

b

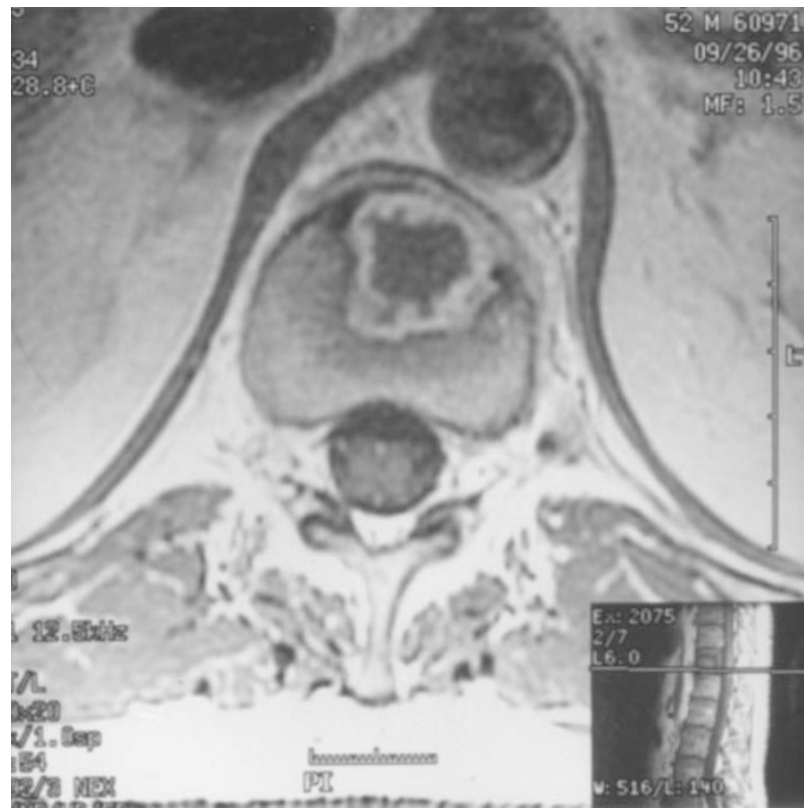

Figure 1 (a) MRI of thoracic spine, T-2 weighted, sagittal section shows the hyperintense lesion of the vertebral body with hypointense rim. Note the preservation of height of the vertebral body and the intact disc spaces. (b) MRI of thoracic spine, T-1 weighted, contrast (Gd-DTPA) enhanced axial image demonstrates the dense rim enhancement of the lesion with central hypointensity. The periphery of the lesion is well demarcated and the prevertebral fascia is also intact

graphic appearance. ${ }^{3}$ Lichtenstein and Jaffe coined the term eosinophilic granuloma in 1940, but later Lichtenstein introduced the term histiocytosis-X; EG being a localized form. Eosinophilic granuloma, a

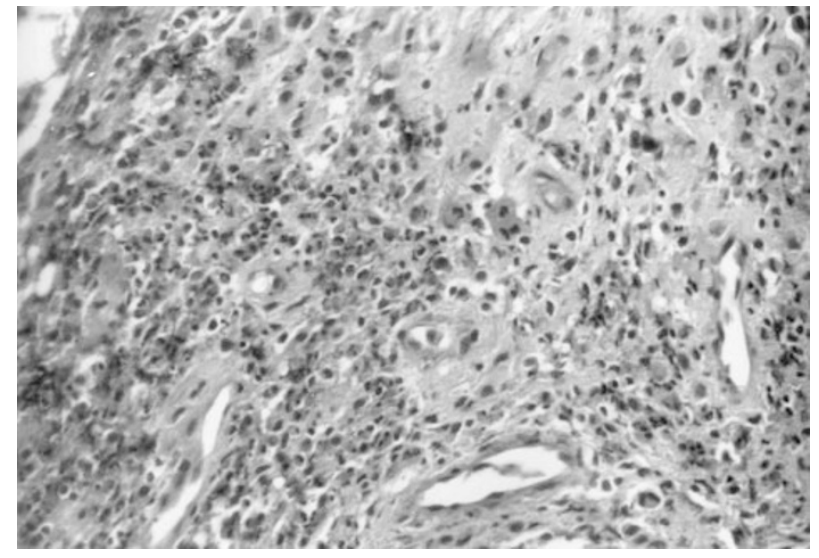

Figure 2 Microphotograph of the lesion from vertebral body reveals proliferated Langerhans giant cells (large clear cells) and numerous eosinophils (H\&E stain; original magnification $\times 250$ )

relatively uncommon disease, accounts for less than $1 \%$ of all bone tumors, but constitutes about $75 \%$ of all cases of Langerhans' cell histiocytosis reported. ${ }^{3}$ Vertebral body involvement is seen $7-15 \%$ of $\mathrm{EG}$ cases. $^{4}$ Approximately $80 \%$ of patients present before age 10, making this generally a disease of childhood. Only 14 cases of EG affecting the adult population have been reported in the literature (Table 1).

Although a disease primarily of bone, established sites for EG include, the skin, gingiva, salivary glands, lung, liver, and lymph nodes. ${ }^{5}$ Clinically, patients with EG of the spine complain of localized pain, decreased range of motion, swelling, and stiffness in their backs. Neurological deficits are not common. ${ }^{6}$ If they occur they are thought to be caused by compression of the spinal neural elements secondary to vertebral collapse or from peridural spread of the disease. ${ }^{7}$

These lesions of eosinophilic granuloma are most frequently evident in the medulla of the bone involved, but lesions may arise in the cortex or in the posterior elements, especially in adults. ${ }^{5} \mathrm{EG}$ is an infiltrative osteolytic process that produces collapse of vertebral bodies, especially in children, and can cause neurological deficit. The radiographic appearance of this vertebral body collapse was termed vertebra plana by Buchman. This classical radiographic finding is seen in $40 \%$ of cases involving children. ${ }^{8}$ In adults, however, relatively small areas of the vertebral body are affected with no associated vertebral collapse. ${ }^{3}$ Villas et al found no relationship between patient age and the development of vertebra plana. However, this was not a radiological finding in adults in any of the cases reported (Table 1).

The level of spinal involvement also differed by age groups. In children, the thoracic spine is the common location for EG $(54 \%)$, followed by the lumbar $(35 \%)$, and cervical spine $(11 \%)$. However, in adults, $47 \%$ of the reported cases had cervical spine involvement, $33 \%$ involved the thoracic spine and the lumbar spine 
Table 1

\begin{tabular}{|c|c|c|c|c|c|c|c|}
\hline Author & Year & Age/Sex & Spine & Neural deficit & $V$. plana & Treatment & Result \\
\hline Dickinson & 1991 & $33 / F$ & $\mathrm{C} 2$ & No & No & $\mathrm{RT}$ & Good \\
\hline Sanchez & 1984 & $55 / \mathrm{F}$ & C6,7 & Yes & No & $\mathrm{S}+\mathrm{RT}+\mathrm{CT}$ & Unchng \\
\hline Martin & 1985 & $31 / \mathrm{F}$ & $\mathrm{C} 5,6$ & Yes & No & Not known & Good \\
\hline Johnson & 1993 & 29/M & $\mathrm{C} 2$ & Yes & No & $\mathrm{S}$ & Not rptd \\
\hline Casson & 1981 & $58 / \mathrm{F}$ & $\mathrm{C} 5$ & Yes & No & $\mathrm{S}$ & Good \\
\hline Acciarri & 1992 & $44 / \mathrm{M}$ & $\mathrm{T} 1$ & Yes & No & $\mathrm{S}+\mathrm{RT}$ & Good \\
\hline Kanterewicz & 1988 & $38 / \mathrm{F}$ & L1 & Yes & No & $\mathrm{B}+\mathrm{RT}+$ Steroids & Good \\
\hline Padovani & 1988 & $20 / \mathrm{M}$ & $\mathrm{T} 12$ & Yes & No & $\mathrm{S}+\mathrm{RT}$ & Good \\
\hline Kaye & 1969 & $21 / F$ & $\mathrm{~L} 2$ & No & No & RT & Good \\
\hline Gibson & 1963 & $33 / \mathrm{M}$ & $\mathrm{T} 10,11$ & Yes & No & $\mathrm{S}$ & Good \\
\hline MGH & 1954 & $35 / \mathrm{M}$ & T11 & Yes & No & $\mathrm{S}+\mathrm{RT}$ & Good \\
\hline Herring & 1987 & $20 / \mathrm{M}$ & $\mathrm{C} 2$ & No & No & $\mathrm{S}+\mathrm{RT}$ & Good \\
\hline Lauffenburger & 1995 & $35 / \mathrm{F}$ & $\mathrm{C} 3$ & No & No & $\mathrm{S}+\mathrm{RT}$ & Good \\
\hline Bilge & 1995 & $34 / \mathrm{F}$ & L5 & No & No & S & Good \\
\hline Present & 1998 & $47 / \mathrm{M}$ & $\mathrm{T} 11$ & No & No & $\mathrm{S}$ & Good \\
\hline
\end{tabular}

$\mathrm{B}=$ biopsy; $\mathrm{CT}=$ chemotherapy; $\mathrm{RT}=$ radiotherapy; $\mathrm{S}=$ surgery; $\mathrm{MGH}=$ Case records of the Massachusetts General Hospital: Weekly clinicopathological exercise

was the site in $20 \%$. CT and MRI have been used for more precise definition of extent of the disease, although no pathognomonic finding has been described.

The differential diagnoses include aneurysmal bone cyst, osteomyelitis, Ewing's sarcoma, osteoblastoma, Gaucher's disease, acute leukemia, a metastatic tumor and a tuberculoma.

Because of the considerable overlap in the clinical and radiological features of these conditions, the diagnosis of EG is dependent on its histological and histochemical identification. Grossly, the tumors are a reddish brown to yellow fibrotic or granular mass interrupted by bony spicules. ${ }^{9}$ The tumors are formed by an aggregate of histiocytes and multinucleated giant cells. Electron microscopy may show Birbeck's granules in the cytoplasm of histiocytes. Immune histological staining for S-100 protein found on the Langerhans' histiocytes is diagnostic of the condition.

A variety of therapeutic approaches have been used either singly or in combination in the treatment of EG and there is a distinct lack of consensus. The approaches include surgical curettage, wide excision and biopsy, local irradiation, systemic chemotherapy, and intra-lesional methylprednisolone injection. ${ }^{10}$ There was no statistically significant difference in the bone healing when these treatment modalities were compared. ${ }^{11}$ Because of its tendency for spontaneous resolution and its self-limiting nature, therapeutic conservatism is encouraged. ${ }^{12}$ The main therapeutic goals should include restoration of bony architecture, reversal of neurological deficit and relief of pain. Indications for surgery include a neurological compromise, instability of spine and/or inconclusive diagnostic procedures.

In summary, we present an eosinophilic granuloma involving the thoracic spine in an adult. An excisional biopsy resulted in symptomatic relief and established the diagnosis. EG rarely involves the adult spine and should be considered in the differential diagnosis of neck and back pain, with osteolytic lesions of the vertebrae. The prognosis is usually excellent in the absence of systemic manifestations. Good healing and recurrence-free survival have been reported with or without radiotherapy.

\section{References}

1 Nishio $\mathrm{S}$ et al. Isolated histiocytosis $\mathrm{X}$ of the pituitary gland: Case Report. Neurosurgery 1987; 21: 718-721.

2 Stull MA, Kransdorf MJ, Devaney KO. Langerhans cell histiocytosis of bone. Radiographics 1992; 12: $801-822$.

3 Sweasey TA, Dauser RC. Eosinophilic granuloma of the cervicothoracic junction. J Neurosurg 1989; 71: 942-944.

4 Cadandell $\mathbf{J}$ et al. Vertebral eosinophilic granuloma; long term evaluation of a case. Spine 1986; 11: 767-769.

5 Huvos AG. Bone tumors. Diagnosis, treatment and prognosis. 2nd edn. Philadelphia: WB Saunders, 1990

6 Lazio BE, Stambough JL. An unusual posterior element spine tumor. Orthopedics 1994; 17: 725-728.

7 Bilge T, Barut S, Yaymaci Y, Alatli C. Solitary eosinophilic granuloma of the lumbar spine in an adult. Case report. Paraplegia 1995; 33: $485-487$.

8 Floman Y et al. Eosinophilic granuloma of the spine. $J$ Paed Orthop 1997; 6-B: $260-265$.

9 Mackenzie WG, Morton KS. Eosinophilic granuloma of bone. Can J Surg 1988; 31: 264-267.

10 Mirra J. Bone tumors: diagnosis and treatment. Philadelphia: Lippincott, 1980.

11 Lauffenburger MD, Dull ST, Toselli R. Eosinophilic granuloma of the adult spine: A case report and review of the literature. $J$ Spinal Disord 1995; 8: $243-245$.

12 Villas C, Martinez-Perric R, Barrios RH, Beguiristain JL. Eosinophilic granuloma of the spine with and without vertebra plana: Long term follow-up of six cases. J Spinal Disord 1993; 6: 260-268. 